Available online at GSC Online Press Directory

GSC Biological and Pharmaceutical Sciences e-ISSN: 2581-3250, CODEN (USA): GBPSC2

Journal homepage: https://www.gsconlinepress.com/journals/gscbps

(RESEARCH ARTICLE)

\title{
High faecal carriage of multi-drug resistant Enterobacteriaceae and other gram negative strains among food handlers in Cosmopolitan Cities of The Gambia
}

\author{
Bakary Sanneh ${ }^{1,}{ }^{*}$, Ollie Diane Riley ${ }^{1}$, Haruna S. Jallow ${ }^{1}$, Abou Kebbeh ${ }^{1}$, Yaya Camara ${ }^{2}$, Ebrima Barrow ${ }^{3}$, \\ Aida Fatou Ceesay ${ }^{1}$, Modou Lamin Jarju 1, Kalifa Sanneh 1, Sainey B.F. Ceesay ${ }^{1}$, Amadou Woury Jallow ${ }^{2}$, \\ Alpha A Jallow ${ }^{4}$, Ignatius Baldeh ${ }^{1}$, Sana M Sambou ${ }^{2}$ and Antoine Andremont 5 \\ ${ }^{1}$ National Public Health Laboratories, Bertil Herding High way, Kotu Lay out, Ministry of Health. \\ 2 Epidemiology and Disease Control Unit, Bertil Herding High way, Kotu Lay out Ministry of Health, The Gambia. \\ 3 Microbiology Laboratory department, Edward Francis Small Teaching Hospital ,Banjul, Ministry Of Health, The Gambia. \\ ${ }^{4}$ World Health Organization, Country Office, Kotu Lay out, The Gambia. \\ ${ }^{5}$ University Paris-Diderot Medical School, 5 Rue Thomas Mann, Paris, France.
}

Publication history: Received on 09 November 2020; revised on 15 November 2020; accepted on 16 November 2020

Article DOI: https://doi.org/10.30574/gscbps.2020.13.2.0364

\begin{abstract}
The introductions of advanced antibiotics have triggered complex versions of resistant genes in gram-negative bacteria. In a generation witness limited discovery of antibiotics makes this phenomenon a major public health threat. This study aims to establish the fecal carriage rate of multidrug-resistant Enterobacteriaceae strains among food handlers.

Data was extracted from a prospective cross-sectional study - 'Prevalence and risk factors of fecal carriage of Extended Spectrum $\beta$-Lactamase producing Enterobacteriaceae amongst food handlers in Lower Basic Schools in West Coast region of The Gambia. The study enrolled 600 randomly selected food handlers (565 of which had complete data set) from 60 lower basic schools in 7 districts in the West Coast Region of The Gambia. Stool samples were collected from the participants and screened for resistance to cefotaxim. Isolated organisms were further tested for susceptibility to cephalosporins, carbapenems, fluoroquinolones and other families of antimicrobials as per Clinical Standard Laboratory Institute guidelines. The association of risk factors to fecal carriage of resistant Enterobacteriacea was performed using Pearson Chi squared and fishers exact $(\mathrm{P}<0.05)$.
\end{abstract}

Results confirmed that the prevalence of Enterobacteriaceae by resistance to cefotaxime was $15.8 \% .23$ genus and 89 species of resistant strains were isolated. All isolates were $100 \%$ resistant to cephalosporins such as ceftriazone, cefotaxime and cefuroxime, except ceftazidime (92\%). Resistance to ciprofloxacin (fluoroquinolone) was measured at $50 \%$. Only $1.2 \%$ was resistant to imipenem (carbapenem). Most isolates were resistant to ampicillin, nitrofurantoine, erythromycin, tetracycline and cotrimaxole. Majority of the identified Enterobacteriaceae were Enterobacter aerogene (14\%), Klebsiella pneumoniea (12\%) and Escherichia coli (7\%).

This study found a high prevalence of fecal carriage multi-drug resistant Enterobacteriaceae among food handlers, mostly women, in The Gambia. The irrational prescription and use of antibiotics is a major risk factor for the proliferation of antibiotic resistance. Well-conducted surveillance is essential to implement effective control measures for the spread of antibiotic resistance urgently receommended.

Keywords: Fecal Carriage; Multi-Drug Resistant; Enterobacteriaceae; Food Handlers; The Gambia

\footnotetext{
${ }^{*}$ Corresponding author: Bakary Sanneh

National Public Health Laboratories, Bertil Herding High way, Kotu Lay out, Ministry of Health. 


\section{Background}

Antimicrobial resistance has become a major threat to the control of infectious diseases and to public health. The limited discovery of antibiotics makes this phenomenon all the more alarming. In places with limited diagnostic facilities and treatment options, it is a major contributing factor to morbidity and mortality [7]. Resistant Enterobacteriaceae strains have been considered to be the most prevalent causative agents of hospital - and community-acquired infections [8]. Multi-resistant strains have been detected in foods and food animals. Therefore, it has been hypothesized that foods are probably one of the most important vehicles for disseminating resistant bacterial strains within the general population [9].

Selective pressure mediated by the intensive use of antibiotics, both human and non-human, contributes to the community's rapid dispersal of antibiotics. This includes antibiotics given empirically without proper antibiotic susceptibility testing [2]. In the hospital setting, outbreaks of Enterobacteriaceae resistant strains have been associated with long term antibiotic exposure, prolonged intensive care unit (ICU) stay, nursing home residency, severe illness, residence in an institution with high rates of ceftazidime and other third generation cephalosporin use and instrumentation or catheterization [4]. Contaminated environments, together with poor sanitation, crowded living conditions and unsafe drinking water supply, in turn, favor the spread of antimicrobial-resistant bacteria through-out the community. Moreover, many developing countries' economies rely on agriculture and livestock keeping, activities known to use large amounts of antimicrobials to increase productivity. This together with the lack of financial resources and political will to address the problem, disastrously made developing countries fertile lands for the evolution of antimicrobial resistance [10].

Studies show that Klebsiella pneumoniae and Escherichia coli are the most common producers of resistant genes and are often implicated among patients with Urinary Tract Infection (UTI). UTIs are among the most common infectious diseases and are usually associated with significant morbidity and mortality. Fecal E. coli is an indicator of the spread of resistant genes in the community. Whereas Klebsiella species are opportunistic pathogens that play significant roles in hospital-acquired infections. Though components of the normal flora, E. coli and Klebsiella species are potential causes of both community and hospital acquired infections [7]. Resistance amongst commensal flora is a serious threat because asymptomatic carriers in the community are often colonized with resistant bacteria that can subsequently lead to extra intestinal infection [2].

There is limited data on antimicrobial resistance of Enterobacteriaceae in The Gambia. Knowledge on the burden and antimicrobial susceptibility pattern of the Enterobacteriaceae isolates is essential for appropriate therapy. This study aims to gain some insight into the antibiotic sensitivity of Enterobacteriaceae in the Gambia, using bacterial isolates obtained from stool samples of food handlers in lower basic schools in the West Coast Region of the Gambia.

\section{Methods}

\subsection{Study design, setting and population}

Data was extracted from the following cross-sectional prospective study: Prevalence and risk factors of fecal carriage of Extended Spectrum $\beta$-Lactamase producing Enterobacteriacea amongst food handlers Lower Basic Schools in West Coast Region of The Gambia[11].

The parent study enrolled 600 food handlers 565 of which had complete data set) from 60 lower basic schools in 7 districts in the West Coast Region of The Gambia. The Lower Basic Schools were grouped into two strata (educational region) as per Ministry of Basic Education (MoBSE) guideline. A random selection of 30 schools in each stratum was then carried out in order to meet a 95\% confidence interval of school population and a sample size of 600[11].

\subsection{Data Collection and Sample Procedure}

Nine (9) food handlers were randomly selected from each school by the social mobilization team made up of staff from the Directorate of Health Promotion and Education, Food Safety and Quality Authority and the Ministry of Basic and Secondary Education. A school with less than 9 food handlers was combined with a nearby school to meet the minimum required number of food handlers [11].. A standardized interviewer-administered questionnaire was used to collect data on socio-demographics informations medical history and risk factors for the potential carriage of antibioticresistant Enterobacteriaceae[11]. 
Stool samples were collected from each participant in well-labeled, sterile and transparent leak-proof plastic containers with screw caps. The field staff immediately recorded the samples' ID and stored at a cold chain temperature for delivery within 6 hours of collection.

The time and temperature at which the samples were collected were signed by the laboratory technician receiving the sample on the custody form chain. The stool samples processing time and date were recorded per batch on the sample processing laboratory logbook. Laboratory Investigation

\subsection{Isolate Identification}

The stool samples were transported and processed in the Microbiology lab within $6 \mathrm{~h}$ of collection. Each stool sample was homogenized in peptone water and aliquots of $0.1 \mathrm{~mL}$ were streaked directly on chromogenic agar (Drigalski agar \& $2 \mathrm{mg}$ CTX). Plates were incubated at $37^{\circ} \mathrm{C}$ and checked for bacterial growth after $24 \mathrm{~h}$. Suspected Enterobacteriaceae isolates were first identified by their colony morphology. Isolated colonies were purified by culture on nutrient agar for confirmation and identification at the species level using Api 20E system [11].

\subsection{Antimicrobial Susceptibility Testing (AST)}

Bacterial susceptibility of all isolates to antibiotics was determined by double disc diffusion method and interpreted following Clinical Laboratory Standard Institute (CLSI) recommendations for Enterobacteriaceae. Pure culture of resistant strains were prepared to $0.5 \mathrm{McFarland}$ turbidity standards in $0.85 \%$ saline and inoculated by spread using sterile swabs on Muller Hinton Agar media as recommended by the Antibiogram Committee of the French Microbiology Society (ACFMS) [3]. The following antibiotics: ceftriazone (CRO), cefotaxime (CTX) and cefuroxime (CRX), ceftazidime (CAZ), ciprofloxacin (CIP) imipenem (IMI) (carbapenem) ampicillin (AMP), nitrofurantoine (NIT), erythromycin (ERY), tetracycline (TET), cotrimaxole (COT) and cefoxitin (FOX) as recommended by CLSI (2015) [[11].The plates were then incubated aerobically at $37^{\circ} \mathrm{C}$ for 24 hours. Klebsiella pneumonia ATCC 700603 (positive control) and Escherichia coli strain ATCC 25922 (negative control) were used as control strains for susceptibility studies as recommended by CLSI guidelines for Enterobacteriaceae[11]. CLSI interpretative criteria were applied for classification of the isolates into susceptible, intermediate and resistant categories using breakpoints for 11 antibiotics. Intermediate and susceptible isolates were considered non-resistant. The multi drug resistant (MDR) phenotype was defined as the demonstrated resistance to at least three antibiotics belonging to different classes of antimicrobial compounds [11].

\subsubsection{Detection of ESBL/AmpC/Carbapenemase production (Phenotypic Tests)}

The ESBL phenotype was determined using cefotaxime and ceftazidime alone, and in combination with a beta-lactamase inhibitor (clavulanic acid). ESBL production was determined as a zone of inhibition of cefotaxime and or ceftazidime more than or equals to $5 \mathrm{~mm}$ from that of cefotaxime/clavulanic acid and or ceftazidime/clavulanic acid[11].Detection of AmpC-type beta-lactamases was performed by testing the bacterial culture for susceptibility to cefoxitin. Resistance to cefoxitin indicated the presence of an AmpC-type beta-lactamase. Confirmatory test for carbapenemase production required the testing of imipenem. Resistance to imipenem indicated that the bacterial strain is a carbapenemaseproducer. Klebsiella pneumonia ATCC 700603 (positive control) and Escherichia coli strain ATCC 25922 (negative control) were used for quality control [CLSI-2015] [11].

During the interpretation of results, strains were categorized into three categories: resistant (R), intermediate (I) and susceptible. All strains susceptible to cefotaxime (CTX), ceftazidme (CAZ), and/or ceftriaxone (CRO) were tested for AmpC, ESBL- or carbapenemase-production by confirmatory tests. Reduced susceptibility to any of the abovementioned antimicrobials indicates that the bacterial strain is an AmpC, ESBL- or carbapenemase-producing phenotype[11].

\subsection{Statistical Analysis/Data Analysis}

Data entry and analysis were performed using SPSS and Epi Info. Results were presented through tables and graphs. Association of risk and socio-demographic factors to the fecal carriage of resistant Enterobacteriaceae strains was measured using Pearson Chi-square and Fisher's exact tests. P-values $<0.05$ were considered to be statistically significant.

\subsection{Ethical Clearance}

The study was approved by the Gambia Government and Medical Research Council Joint Ethical Committee with number SCC1416 of the main study where this data is extracted [11]. Informed consent was obtained from each study participant by request of their signature for storage of sample,data to support order study. 


\section{Results}

\subsection{Socio-demographic factors of food handlers}

A total of 600 food handlers were enrolled in the study and 565 study participants with complete data set were included in the analysis. $99.3 \%$ of food handlers were female with a mean age of 37yrs. Most of the study participants stayed less than $5 \mathrm{~km}$ from the nearest health facility. , The majority of food handlers, have been selling and preparing food for not more than 4yrs and have been certified. Only $17 \%$ of food handlers have been trained on food safety procedures and a high percentage of $91.2 \%$ acclaimed to know the basic principles of food safety as shown in table 1.

Table1 Socio-demographic factors of food handlers

\begin{tabular}{|l|l|l|l|}
\hline Characteristics & Number & Percentage (\%) & P-value \\
\hline Mean age (yrs ) & 37 & & 0.112 \\
\hline Gender (female) & 561 & 99.3 & 0.452 \\
\hline Live <5km from nearest health facility & 551 & 98.5 & 0.698 \\
\hline Rare domestic animals & 351 & 62.1 & 0.569 \\
\hline Selling food for $\leq 4 \mathrm{yrs}$ & 409 & 72.38 & 0.24 \\
\hline
\end{tabular}

\subsection{Geographical distribution and prevalence MDR carriage}

The prevalence of the fecal carriage of Enterobacteriaceae strains was $15.8 \%(89 / 565)$. Region one, represented by KMC, recorded a 49\% (44/89) prevalence rate of antibiotic-resistant carriers. Region two recorded a prevalence rate of $51 \%(45 / 89)$ as shown in table 2. In the region, Kombo North recorded a high rate of 30.34\% (27/89), followed by Kombo South, 12.36 (11/89). Foni Bintang and Foni Kansala had 2 carriers each (2.25\%). The remaining districts (Kombo Central, Foni Berefet, and Foni Jarrol) only had 1 carrier each (1.12\%). except Kombo East district where no antibiotic resistant Enterobacteriaceae strains were found among food handlers as shown in table 2.

Table 2 Geographical distribution and prevalence of MDR carriage in food handlers

\begin{tabular}{|l|l|l|}
\hline \multicolumn{3}{|l|}{ AMR AMONG DISTRICTS } \\
\hline Districts & Number & Percentage (\%) \\
\hline KMC & 44 & 49.44 \\
\hline Foni Bintang & 2 & 2.25 \\
\hline Foni Berefet & 1 & 1.12 \\
\hline Foni Jarrol & 1 & 1.12 \\
\hline Foni Kansala & 2 & 2.25 \\
\hline Kombo North & 27 & 30.34 \\
\hline Kombo Central & 1 & 1.12 \\
\hline Kombo South & 11 & 12.36 \\
\hline Kombo East & 0 & 0 \\
\hline
\end{tabular}

A total 23 genuses and 89 species of resistant Enterobacteriaceae strains were isolated. Majority of the identified Enterobacteriaceae species were Enterobacter aerogene (13.50\%), Klebsiella pneumoniea (13.50\%) and Escherichia coli $(9 \%)$ as in table 3 . 
Table 3 Distribution of resistant strains by species

\begin{tabular}{|c|c|c|c|c|c|c|c|c|c|c|c|}
\hline Isolates & \begin{tabular}{|l|} 
CTX \\
$30 \mu \mathrm{g}$
\end{tabular} & $\begin{array}{l}\text { CRO } \\
30 \mu \mathrm{g}\end{array}$ & $\begin{array}{l}\text { CAZ } \\
30 \mu \mathrm{g}\end{array}$ & $\begin{array}{l}\text { FOX } \\
30 \mu \mathrm{g}\end{array}$ & $\begin{array}{l}\text { IMI } \\
10 \mu \mathrm{g}\end{array}$ & $\begin{array}{l}\text { NIT } \\
300 \mu \mathrm{g}\end{array}$ & $\begin{array}{l}\text { CIP } \\
10 \mu \mathrm{g}\end{array}$ & $\begin{array}{l}\text { ERY } \\
10 \mu g\end{array}$ & $\begin{array}{l}\text { AMP } \\
10 \mu \mathrm{g}\end{array}$ & $\begin{array}{l}\text { TET } \\
30 \mu \mathrm{g}\end{array}$ & $\begin{array}{l}\text { COT } \\
25 \mu \mathrm{g}\end{array}$ \\
\hline Serratia spp & 2 & 2 & 2 & 1 & 0 & 2 & 1 & 2 & 2 & 1 & 1 \\
\hline Enterobacter spp & 14 & 14 & 13 & 12 & 5 & 8 & 6 & 13 & 14 & 13 & 8 \\
\hline klebsiella pneumonia & 12 & 12 & 12 & 6 & 0 & 8 & 8 & 12 & 12 & 12 & 12 \\
\hline Escherichia coli & 8 & 8 & 8 & 3 & 1 & 6 & 6 & 8 & 8 & 8 & 7 \\
\hline Pseudomonas spp & 13 & 13 & 13 & 10 & 4 & 11 & 4 & 12 & 11 & 11 & 8 \\
\hline Citerobacter spp & 4 & 4 & 4 & 2 & 0 & 3 & 3 & 4 & 4 & 4 & 3 \\
\hline Yersinia frederiksenii & 1 & 1 & 1 & 1 & 0 & 1 & 1 & 1 & 1 & 1 & 1 \\
\hline kluyvera spp & 3 & 3 & 3 & 3 & 0 & 3 & 0 & 3 & 3 & 3 & 3 \\
\hline Cronobacter spp & 1 & 1 & 1 & 0 & 0 & 1 & 1 & 0 & 0 & 0 & 1 \\
\hline Proteus mirabilis & 1 & 1 & 0 & 1 & 0 & 1 & 1 & 1 & 1 & 1 & 1 \\
\hline Salmonella $s p p$ & 6 & 6 & 6 & 3 & 1 & 5 & 0 & 5 & 6 & 6 & 3 \\
\hline Cedecea spp & 4 & 4 & 4 & 2 & 0 & 1 & 1 & 2 & 2 & 2 & 1 \\
\hline Stenotrophomonas maltophilia & 3 & 3 & 3 & 2 & 1 & 2 & 0 & 3 & 3 & 3 & 0 \\
\hline Burkhole cepacia & 2 & 2 & 2 & 1 & 1 & 1 & 1 & 1 & 1 & 1 & 0 \\
\hline Pantoea spp & 2 & 2 & 2 & 2 & 0 & 2 & 1 & 2 & 2 & 2 & 0 \\
\hline Pasteurella pneumotropical & 2 & 2 & 2 & 2 & 1 & 1 & 1 & 2 & 2 & 2 & 0 \\
\hline Vibrio fluvalis & 5 & 5 & 4 & 2 & 1 & 2 & 1 & 4 & 4 & 3 & 1 \\
\hline Ewingella Americana & 1 & 1 & 1 & 1 & 0 & 1 & 0 & 1 & 1 & 1 & 1 \\
\hline Aeromonas hydrophila & 1 & 1 & 1 & 1 & 1 & 0 & 0 & 1 & 1 & 1 & 1 \\
\hline Erwinia spp & 1 & 1 & 1 & 0 & 0 & 0 & 1 & 1 & 1 & 1 & 1 \\
\hline Ochrobactrumanthropi spp & 1 & 1 & 1 & 1 & 0 & 1 & 0 & 0 & 1 & 1 & 0 \\
\hline Unidentified & 1 & 1 & 1 & 1 & 0 & 1 & 1 & 1 & 1 & 1 & 0 \\
\hline Shigella spp & 1 & 1 & 0 & 1 & 0 & 1 & 0 & 1 & 1 & 1 & 0 \\
\hline
\end{tabular}

\subsection{Antibiotic Resistance Pattern among food handlers}

All fecal bacteria isolates were tested for their susceptibility to 11 antibiotics or antibiotic combinations covering 8 classes of antimicrobial compounds, namely; aminoglycoside, cephalosporin, carbapenem, tetracycline, fluoroquinolone, macrolide, nitrofurantoin, and imidazole. All isolates were $100 \%$ resistant to the third generation cephalosporins such as ceftriazone, cefotaxime and cefuroxime, except ceftazidime (92\%). 50\% of isolates were resistant to ciprofloxacin. Only $1.2 \%$ was resistant to imipenem (carbapenem). Large proportions of the isolates were also found to be resistant to ampicillin (96\%), nitrofurantoin (66\%), erythromycin (96\%), tetracycline (89\%), cotrimaxole (79\%), and cefoxitin (53\%) as in fig1. All of the isolates were MDR (100\%). Escherichia, Enterobacter, Pseudomonas and Vibrio spp. tested resistant to all antibiotics by disk diffusion. E. coli isolates were resistant to all drugs. K. pneumonia isolates were resistant to all antibiotics except imipenem. The lowest level of resistance was displayed by Cronobacter and Ochrobactrumanthropi spp shown in fig 1. 


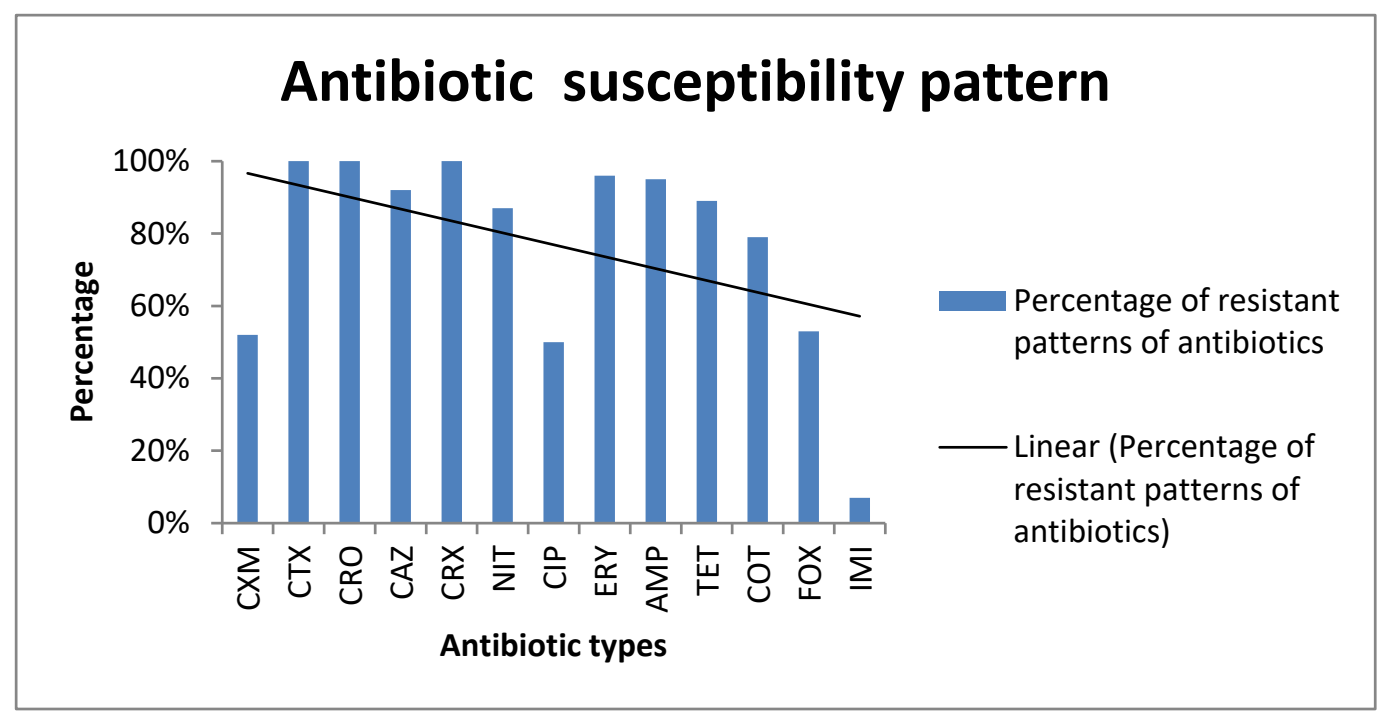

Figure 1 Antibiotic Resistance Pattern among food handlers

\subsubsection{Phenotypic distribution of resistant strains}

Resistance to ceftazidime is indicative of ESBL production but may also be due to inducible AmpC enzyme common in Enterobacter species. Resistance to cefoxitin indicated the presence of an AmpC-type beta-lactamase. The tests revealed that $20.22 \%$ (18/89) of the total number of isolates were phenotypically classified as ESBL producers as defined by CLSI criteria. 46.07\% (41/89) of the strains were found to be AmpC-producing strains. 20.22\% (18/89) were found to be both ESBL and AmpC producers (coproduction). Only 1.12\% (1/89) of the strains was resistant to imipenem and consequently classified as carbapenemase as shown in fig 2.

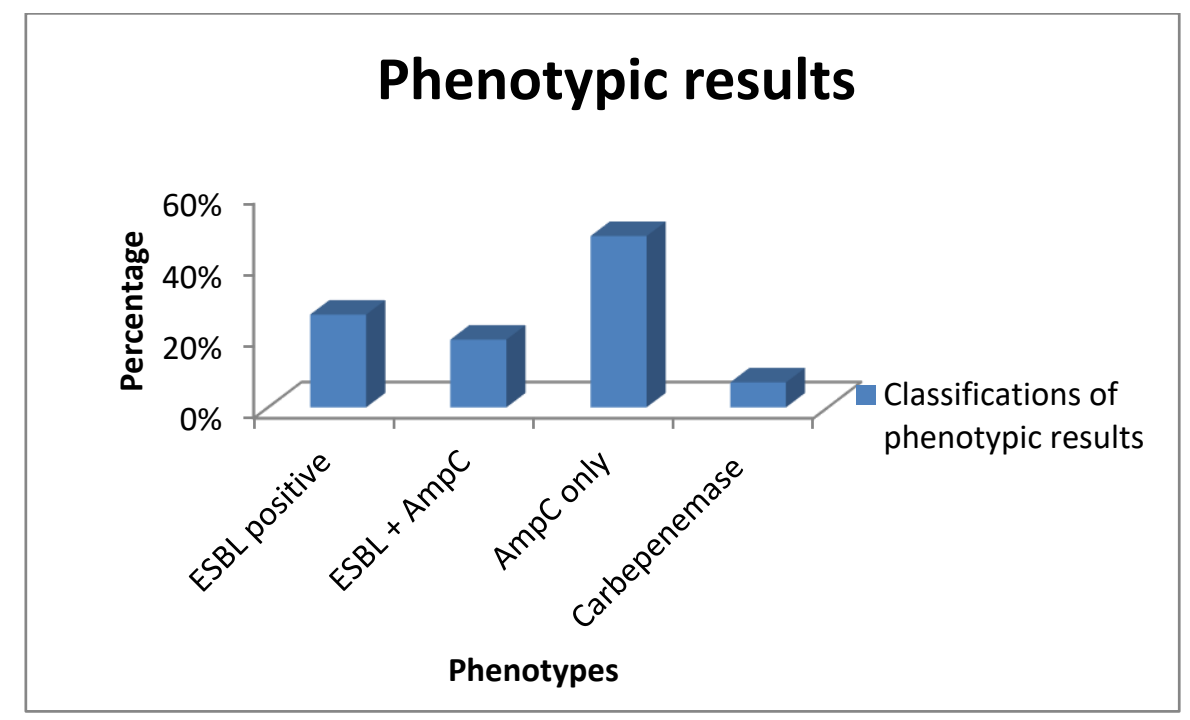

Figure 2 Phenotypic distribution of resistant strains among food handlers

\subsubsection{Risk factors for carriage of multidrug resistant bacteria}

Lack of food handling training and food safety principles are strongly associated with AMR carriage with P-values of 0.001 and 0.002 , respectively. The use of antibiotics in the last 3 months has shown associated risks to this multi-drugresistant Enterobacteriaceae carriage. Other risks of the same evidence of inference for the carriage of resistance include diarrheal episodes in the past three months, $(\mathrm{p}=0.042)$ and compliance to completion of prescribed antibiotics $(\mathrm{p}=0.0555)$. Other identified risk factors were found to the insignificantly associated with the carriage of resistant strains as in Table 4. 
Table 4 Risk factors for carriage of multidrug resistant bacteria among food handlers

\begin{tabular}{|c|c|c|c|}
\hline Risk Factor & $\begin{array}{l}\text { Number of } \\
\text { Respondents }\end{array}$ & Percentage (\%) & P-value \\
\hline Lacks food handling training & & & 0.001 \\
\hline Lacks knowledge on food safety principles & & & 0.002 \\
\hline Wash hands with soap under running water & 560 & 99.1 & 0.385 \\
\hline Use pit latrine toilet facilities & 329 & 58.3 & 0.325 \\
\hline Admitted in hospital in the past 3 months & 13 & 2.3 & 0.134 \\
\hline Used medical instrumentation while admitted & 4 & 0.71 & 0.385 \\
\hline $\begin{array}{l}\text { Escorted /visited a sick family member to the hospital in the } \\
\text { past three months }\end{array}$ & 84 & 14.9 & 0.934 \\
\hline $\begin{array}{l}\text { Stayed with somebody who had been admitted in the } \\
\text { hospital }\end{array}$ & 89 & 15.8 & 0.741 \\
\hline Used antibiotics in the past three months & 185 & 32.7 & 0.012 \\
\hline Bought and used antibiotics from street vendors & 17 & 3 & 0.83 \\
\hline $\begin{array}{l}\text { Used antibiotics without consultation from a medical } \\
\text { practitioner }\end{array}$ & 13 & 2.3 & 0.418 \\
\hline Completed antibiotic dosage & 545 & 96.4 & 0.055 \\
\hline Had diarrhea in the past three months & 68 & 12.03 & 0.042 \\
\hline Had urinary tract infection in the past three months & 13 & 2.3 & 0.968 \\
\hline
\end{tabular}

\section{Discussion}

This study aimed to evaluate the intestinal carriage rate of Enterobacteriaceae resistant strains and create awareness of multi-drug resistance. It confirms the presence of high multidrug resistance among gram-negative Enterobacteriaceae in the country. The highest prevalence was found in the cosmopolitan district of Kanifing Municipal Council (KMC), a buzzing district with the most basic amenities. Findings, therefore, prove that MDR carriers are common in cosmopolitan districts. Very few cases were found in remote districts which could perhaps be associated to less access to antibiotic and high rate of poverty in such communities. They are also the communities with much less public and private facilities dispensing antibiotics. Further study is recommended to better understand this phenomena.

Overall, Klebsiella pneumoniae, E. coli and Enterobacter aerogenes account for most of the isolated resistant bacteria species. These results are identical with a study conducted by Andriatahina et al.(2010) in Madagsacar, where the above mentioned species were the most dominant.In contrast, the study Leski et al. conducted in Sierra Leone on resistant bacterial strains using urine isolates (2016), showed a higher prevalence of Citrobacter freundi. Though isolated in small quantities in our study sample (3.37\%), Citrobacter indicates an increasingly important emerging urinary tract pathogen. Most isolates showed similar resistance patterns, sensitive to imipenem while insenstive to third generation cepholosporins. K. pneumoniae isolates were significantly less resistant to imipenem than E. coli (P-value, percentages)

Leski et al. (2016) discovered an 85.7\% MDR rate, 64.3\% of which were ESBL producers. Our study however discovered a higher rate of MDR (100\%) but a lower rate of total ESBL production (40.45\%). In their study, $C$. freundi exhibited the highest level of MDR while E. coli exhibited the lowest. The opposite was true for us, with E. coli demonstrating one of the highest levels of resistance and $C$. freundi one of the lowest.

The analysis of stool samples from food handlers shows the prevalence of clinically relevant MDR bacteria in the community, confirmed by phenotypic assay. Especially alarming is the fact most strains were not only resistant to firstline antibiotics such as beta-lactams but to second-line drugs as amino-glycosides and broad spectrum antibiotic. 
Particularly worrisome is that even normal flora E. coli, which is usually less multi resistant, was found to be significantly MDR. With Kothari and partners, ESBL, AmpC and coproduction was seen in 20.6\%, 19.9\% and 11.2\% isolates respectively. Similarly in our study, ESBL was seen in $20.22 \%$ isolates but AmpC and coproduction was seen in $46.07 \%$ and $20.22 \%$ isolates, respectively.Studies done at the Edward Francis Small Teach Hospitals of the Gambia had also reported increased antibiotic resistance trends among Enterobacteriacea notably $K$. pneumoniae and E. coli and had revealed the high level of patients been treated with antibiotic [12,13].These increases the of transmission of transmission of such antibiotic bacterium in the country in either the hospital or community setting.

In univariate analysis, significant risk factors for MDR carriage were prior use of antibiotic, diarrheal episodes 3 months prior to the study and lack of knowledge in food safety practices. Interestingly in our study, hospital admission, instrumentation while on admission, and staying with a previously hospitalized individual were not significantly associated with MDR carriage, as opposed to other studies [3]. Demographic characteristics showed no significant association to MDR carriage. Inability to complete prescribed drugs had some inference to the carriage of these gram negative pathogens, Raring of domestic animals and distance to with the nearest health facility were also not significantly associated with resistance carriage. Episodes of UTI are dependent risk factors to the carriage of ESBL. We found that use of antibiotic in the past 3 months was significantly associated with the carriage of multidrug resistance bacteria. Therefore the more Gambian population are been exposure to antibiotic treatment so more we are risk of the development of antibiotic resistance bacteria and their spread in both communities and hospitals has been alluded in other national studies [12,13]. A based study on AMR stewardship among health workers nationally found majority of the workers are aware of the increase and reasons for antibiotics yet some of them still prescribed antibiotic for viral related infections [14].Thus the urgent need for the development of national policies and strategic plans for the control of the spread of antibiotics.

\section{Limitation}

Most of the Lower Basic Schools were located in Kanifing Municipal Council which might forms the bias in the distribution ESBLs at district level. Not all the isolated Enterobacteriaceae could have been speciated to better understand the spectra of Enterobacteriaceae spp in food handlers. Molecular confirmatory test was not done to establish genotypes ESBLs and AmpC are in circulation in the Gambia. Confirmatory test were not done for the isolates which were resistant to imipenem for cabapenemase phenotypes. The participants were unable to provide their medical record in order to confirm the prescribed antiobiotics. The study was conducted during school holidays and not all food handlers were met for consenting to participants in the study. This MDR prevalence only represents single fraction of the normal population and the actual burden of the infection on the symptomatic patients remain at large.

\section{Conclusion}

This study found a high prevalence of fecal carriage multi-drug resistant Enterobacteriaceae among food handlers, mostly women, in The Gambia who are mainly residing in cosmopolitan cities. These findings concluded that the irrational prescription and use of antibiotics is a major risk factor for the proliferation of antibiotic resistance in cosmosploitan cities. Well-conducted surveillance is essential to implement effective control measures. The study has established the presence of ESBL and AmpC and faecal carriage in the food handlers who as principal in the transmission of potential public health aetiological agents. Irrational usages of antibiotics, lack of knowledge on food safety are major risk factors to the carriage of the ESBL.Antibiotic susceptibility testing results show that clinically relevant MDR Enterobacteriaceae are prevalent in the studied population, which may reflect a lack of antibiotic control policies in the country and widespread access to antibiotics without prescription. International collaboration is needed to help developing countries respond to the threat of infection with multidrug-resistant bacteria.

\section{Compliance with ethical standards}

\section{Acknowledgments}

We wish to thanks all the field workers and appreciated all the food handlers who had participated in the study.We also acknowledge the funding support of World Health Organization,Food Safety Department ,Head Quarter,Geneva for funding the main study from which this paper has been developed.

\section{Disclosure of conflict of interest}

All authors declared there was no conflict of interest. 


\section{Statement of informed consent}

All the study participants had consented to participate in the main study from which the data use to prepare this manuscript had been extracted.

\section{References}

[1] Eshetie S, Unakal C, Gelaw A, Ayelign B, Endris M, Moges F. Multidrug resistant and carbapenemase producing Enterobacteriaceae among patients with urinary tract infection at referral Hospital, Northwest Ethiopia. Antimicrobial resistance and infection control. 2015 Dec 1; 4(1):12.

[2] Kothari C, Gaind R, Singh LC, Sinha A, Kumari V, Arya S, Chellani H, Saxena S, Deb M. Community acquisition of $\beta$ lactamase producing Enterobacteriaceae in neonatal gut. BMC microbiology. 2013 Dec 1; 13(1):136.

[3] Andriatahina T, Randrianirina F, Hariniana ER, Talarmin A, Raobijaona H, Buisson Y, Richard V. High prevalence of fecal carriage of extended-spectrum $\beta$-lactamase-producing Escherichia coli and Klebsiella pneumoniae in a pediatric unit in Madagascar. BMC infectious diseases. 2010 Dec 1; 10(1):204.

[4] Yadav KK, Adhikari N, Khadka R, Pant AD, Shah B. Multidrug resistant Enterobacteriaceae and extended spectrum $\beta$-lactamase producing Escherichia coli: a cross-sectional study in National Kidney Center, Nepal. Antimicrobial resistance and infection control. 2015 Dec 1; 4(1):42.

[5] Leski TA, Taitt CR, Bangura U, Stockelman MG, Ansumana R, Cooper WH, Stenger DA, Vora GJ. High prevalence of multidrug resistant Enterobacteriaceae isolated from outpatient urine samples but not the hospital environment in Bo, Sierra Leone. BMC infectious diseases. 2016 Dec 1; 16(1):167.

[6] Guyomard-Rabenirina S, Malespine J, Ducat C, Sadikalay S, Falord M, Harrois D, Richard V, Dozois C, Breurec S, Talarmin A, Laboratory working group. Temporal trends and risks factors for antimicrobial resistant Enterobacteriaceae urinary isolates from outpatients in Guadeloupe. BMC microbiology. 2016 Dec 1; $16(1): 121$.

[7] Najjuka CF, Kateete DP, Kajumbula HM, Joloba ML, Essack SY. Antimicrobial susceptibility profiles of Escherichia coli and Klebsiella pneumoniae isolated from outpatients in urban and rural districts of Uganda. BMC research notes. 2016 Dec 1; 9(1):235.

[8] Ahmed SF, Ali MM, Mohamed ZK, Moussa TA, Klena JD. Fecal carriage of extended-spectrum $\beta$-lactamases and AmpC-producing Escherichia coli in a Libyan community. Annals of clinical microbiology and antimicrobials. 2014 Dec $1 ; 13(1): 22$.

[9] Lavilla S, Gonzalez-Lopez JJ, Miro E, Dominguez A, Llagostera M, Bartolome RM, Mirelis B, Navarro F, Prats G. Dissemination of extended-spectrum $\beta$-lactamase-producing bacteria: the food-borne outbreak lesson. Journal of antimicrobial chemotherapy. 2008 Jun 1; 61(6):1244-51.

[10] Bonelli RR, Moreira BM, Picão RC. Antimicrobial resistance among Enterobacteriaceae in South America: history, current dissemination status and associated socioeconomic factors. Drug Resistance Updates. 2014 Apr 1; 17(12):24-36.

[11] Sanneh B, Kebbeh A, Jallow HS, Camara Y, Mwamakamba LW, Ceesay IF, Barrow E, Sowe F0, Sambou SM, Baldeh I, Jallow A. Prevalence and risk factors for faecal carriage of Extended Spectrum $\beta$-lactamase producing Enterobacteriaceae among food handlers in lower basic schools in West Coast Region of The Gambia. PLoS One. 2018 Aug 13; 13(8):e0200894.

[12] Okomo UA. Neonatal Infections; a hospital-based study in The Gambia examining aetiology and associated maternal Colonisation (Doctoral dissertation, London School of Hygiene \& Tropical Medicine).2018

[13] Chaw PS, Schlinkmann KM, Raupach-Rosin H, Karch A, Pletz MW, Huebner J, Nyan O, Mikolajczyk R. Antibiotic use on paediatric inpatients in a teaching hospital in the Gambia, a retrospective study. Antimicrobial Resistance \& Infection Control. 2018 Dec;7(1):82.

[14] Sanneh B, Jallow HS, Singhateh Y, Sabally B, Sey AP, Jallow AW, Jallow T, Baldeh I, Sambou SM, Jah SL. Knowledge, attitude and practice of health care workers on antibiotic resistance and usage in the Gambia. GSC Biological and Pharmaceutical Sciences, 2020, 13(02), 007-015 